\title{
Exposure and Knowledge of Sharps Injuries among Medical Students in Seven States of Mexico
}

Adrián Camacho-Ortiz, ${ }^{\prime}$ Xiomara Díaz-Rodríguez, ${ }^{2}$ Raúl Hernández-García, ${ }^{2}$ Mayra Martínez-Palomares, ${ }^{2}$ Susana ChávezMoreno, ${ }^{2}$ Elvira Garza-González, ${ }^{3}$ Rafael Valdez-Vázquez, ${ }^{4}$ Irlanda Díaz-Pino. ${ }^{5}$

\begin{abstract}
Background: Medical students are vulnerable to accidental exposure to blood-borne pathogens when performing clinical activities. Knowledge of both the prevalence of exposure and necessary reporting procedures is important to minimize the risk of harm to medical students. Methods: A cross-sectional online survey of medical students from 19 universities from seven states in Mexico was utilized to determine the prevalence of needle stick injuries amongst medical students and the associated reporting procedures. Results: We included 312 respondents; of these, $52.24 \%$ were men and $47.76 \%$ were women, and the mean age was 23.19 years ( $S D \pm 2.11$ years). Nearly all of them (94.23\%) were medical students doing clerkships in public hospitals. Mean knowledge score of blood-borne pathogens was 3.6 (SD \pm 1.16 ) on a scale of 0-10 designed specifically for this study. Thirty-five per cent of the respondents had sustained a needle stick injury at some point during their medical school training, and $33.97 \%$ reported some type of mucocutaneous exposure. Overall, the non-reporting rate of needle stick injury was $48.34 \%$. Approximately $25 \%$ of the respondents were not familiar with reporting procedures in the event of a needle stick injury or mucocutaneous exposure; $61.50 \%$ had received information from their hospital about the standard protocol to follow after a blood or body fluid exposure. Conclusion: In this Mexican population of medical students, there is a high risk of suffering needle stick injuries during medical training. Furthermore, knowledge regarding prevention, evaluation, and reporting of needle stick injuries is suboptimal.
\end{abstract}

Keywords: Needle Stick Injury, Medical Student, Body Fluids, Blood-Borne Pathogens, HBV-ASO4 vaccine, HIV, Preventive Measures (Source: MeSH-NLM).

About the Author: Xiomara S. Díaz-Rodríguez graduated from a 7 year medical school program in 2014 from the Facultad de Me dicina de la Universidad de Nuevo León in Monterrey, México.

\section{Introduction}

Sharps injuries are frequent events among medical students.' As many as one out of three exposed medical students may be at risk of acquiring human immunodeficiency virus (HIV), hepatitis B virus (HBV), or hepatitis C virus (HCV) infection during medical training due to blood or body fluid exposures, with the intern year of training being the most common period in medical training to endure a needle stick injury (NSI) or mucocutaneous exposure. ${ }^{2}$ Under-reporting is common because of the belief that most exposures are not significant, time constraints, and not knowing where to report the event. ${ }^{2-3}$ Furthermore, medical students are more likely to ignore the exposure than residents or fellows. ${ }^{4}$

The National Institute for Occupational Safety and Health (NIOSH) defines NSIs as "those caused by needles such as hypodermic needles, blood collection needles, intravenous (IV) stylets, and needles used to connect IV delivery systems". (Centers for Disease Control and Prevention. Available from: http://www.cdc.gov/niosh/, updated 2015 February 15; cited 2015 February 26.) The risk of NSIs among health care workers is high; this risk can best be reduced by taking proper preventive measures including immunization against $\mathrm{HBV}$, eliminating unnecessary injections, implementing universal precautions, eliminating needle re-capping and disposing of the sharps into a sharps-container immediately after use. Furthermore, utilization of safer devices such as needles that sheath or retract after usage, provision and use of personal protective equipment, and training workers in the risks and prevention of transmission can all reduce the likelihood of NSIs. Post-exposure prophylaxis with antiretroviral medications has been shown to reduce the risk of HIV transmission by $80 \%$ (Centers for Disease Control and Prevention. Available from: http://www.cdc.gov/hicpac/2007lP/2007isolationPrecautions.html, updated 2010 December 9; cited 2015 February 26). ${ }^{5}$

Data regarding the psychological impact of a needle stick or sharps injury is limited when compared to published research describing the physical risk of seroconversion. Healthcare workers who have had a needle stick or sharps injury undergo high levels of anxiety and depression during work, and these are exacerbated after the injury. ${ }^{6}$

The aim of this study is to evaluate the prevalence of NSIs and mucocutaneous exposures, knowledge of blood-borne diseases, and post-exposure actions taken by interns and medical students from different medical schools in Mexico.

\section{Methods}

We performed a cross-sectional, anonymous, open online survey from April to July 2013 that included medical students from 
19 universities from 7 states in Mexico. A total of 400 questionnaires were sent; we included only fully and consistently answered questionnaires. Those questionnaires that had inconsistent answers with discrepancies were eliminated

The questionnaire consisted of two sections with 28 questions covering past experiences of NSIs, mucocutaneous exposures, the circumstances surrounding the injury, reporting protocols, the use of gloves, recapping practices, HBV vaccination, and the perceived level of anxiety after exposure and post-exposure prophylaxis. The respondents were also asked to select from a list of pathogens the ones they thought could be transmitted by blood or body fluid exposure. The list included HIV, HBV, HCV, cytomegalovirus, Epstein Barr Virus, dengue virus, Trypanosoma spp, Mycobacterium tuberculosis, Leishmania spp and Treponema pallidum. Streptococcus pneumoniae and Neisseria meningitidis were added as distracters. Five questions asked for basic demographic data.

Consent was presumed upon submission of the questionnaire. Data was obtained using a Google Survey (Google Incorporated, Mountain View, CA). The online web link was distributed using Facebook (www.facebook.com) and by personal e-mail. Once the questionnaire was constructed, it was randomly applied to a group of 15 medical students for the pre-evaluation of the understanding of the questions. Afterwards, simple modifications were made before applying the survey to the study group. All of the study questionnaires were sent and evaluated by the authors.

We calculated an approximate population of 350,000 students that could potentially be surveyed. Based on this population, we estimated that for a confidence level of $95 \%$, a total of 400 questionnaires would be required. (www.surveysystem.com). All data was analyzed using simple descriptive statistics. The variables were expressed in mean, median and standard deviation. A Chi-squared test was used when comparing proportions and a Wilkinson sum test was used when comparing means. A p-value of, 0.05 was considered to be statistically significant. We used Statistical Package for the Social Science S.P.S.S. version 18 software.

The study was approved by the Ethics Committee on November 22th, 2013 under the approval code IF13-004.

\section{Results}

A total of 338 questionnaires were completed. Twenty-six were eliminated because they had inconsistent answers and discrepancies. We analyzed the data from the remaining 312 respondents. Of these, $52.24 \%$ were from men and $47.76 \%$ from women. Seventy-three per cent of the respondents $(n=228)$ were from Nuevo Leon state, $9.94 \%(n=31)$ from Mexico City, $8.33 \%(n=26)$ from Baja California state, $5.45 \%(n=17)$ from Puebla state, and $2.56(n=8)$ from Jalisco state, and 2 more from other states.

Two-hundred and eight questionnaires (66.9\%) were from the Universidad Autónoma de Nuevo León, $26(8.33 \%)$ from the Universidad Nacional Autónoma de México, 20 (6.41\%) from the Universidad de Monterrey, $15(4.81 \%)$ from the Universidad Xochicalco, 17 from the Universidad Autónoma de Puebla, 8 from the Universidad de Guadalajara, and 18 more from other universities.
A total of 294 students (94.23\%) were doing their clerkships in a public hospital while $16(5.13 \%)$ were assigned to a private hospital at the time of the survey.

The mean student age was 23.19 years (range 20-30 years) (SD +2.11 years). Two-hundred and ninety five $(94.55 \%)$ respondents had had clinical practice in the hospital. Twothirds (218/312) were final year students. Sixty-three percent (199/312) had attended at least one lecture about NSIs and mucocutaneous exposure during their medical education and $56.73 \%$ (177/312) reinforced their knowledge of sharps and mucocutaneous exposure with a lecture before starting their clinical practices.

Students were asked to rate their own knowledge about NSIS and mucocutaneous exposures as bad, poor, regular, good, or very good. Eleven (3.53\%) students rated their knowledge as bad, $26(8.33 \%)$ as poor, $128(41.03 \%)$ as regular, $131(41.99 \%)$ as good, and $16(5.13 \%)$ as very good.

The percentages of students who correctly identified blood-borne pathogens were as follows: One hundred percent $(n=312)$ of the students identified HIV as a blood-borne pathogen, $96.15 \%$ $(n=300)$ identified HBV, 91.98\% $(n=287)$ identified HCV, 32.69\% $(n=102)$ identified cytomegalovirus, $21.47 \%(n=67)$ identified Epstein Barr virus, $9.61 \%(n=30)$ identified dengue virus, $7.69 \%$ $(n=24)$ identified Trypanosoma spp, $21.79 \%(n=68)$ identified M. tuberculosis, $6.73 \%(n=21)$ identified Leishmania spp and $25.96 \%$ $(n=81)$ identified T. pallidum as blood-borne pathogens. One point was added for each correct answer and one was subtracted for each incorrect answer (theoretically, there was a maximum score of 10 and a minimum of o points). In the current study, the mean score was 3.66 (range 3-10) (SD +1.16). When we compared results from students from public universities to private universities, there were no statistical differences $(p=0.97)$.

of the respondents, $68.0 \%$ from public universities and $51 \%$ from private universities stated that they had received information about prevention practices for related injuries during their medical training $(p=0.005)$, demonstrating a significant difference between public and private universities. Thirty-four per cent (106/312) of the respondents had sustained an NSI at some point during their medical school training and $104(33.33 \%)$ sustained some type of mucocutaneous exposure. Seventy-nine students $(25.32 \%)$ had suffered an accident during the six months prior to answering the questionnaire. When comparing medical students who sustained NSIs, $26.9 \%$ belonged to public universities while $38.5 \%$ were from private universities $(p=0.050)$. Almost $90 \%(277 / 312)$ of the respondents knew of at least one fellow student who had had a blood or body fluid exposure. Of the 218 students in their last year of medical studies, $37.15 \%(n=81)$ had sustained at least one NSI. Figure 1 demonstrates the percent of medical students with NSIs according to their year of training.

The overall non-reporting rate of NSIs and mucocutaneous exposures was $48.34 \%(n=73)$. Of the students who reported the incident, $67.95 \%(n=53)$ did it during the first hour post-exposure. Only two of them (2.5\%) reported the incident 48 hours after exposure (Table 1). 
Blood tests for blood-borne pathogens including HIV, HBV and $\mathrm{HCV}$ were performed in both the source patient and the student who reported the accident in $76.92 \%$ and $58.97 \%$ of the events, respectively. Only $8(10.25 \%)$ of the incidents involved a patient already diagnosed with an HIV, HBV, or HCV infection. The source patient could not be identified by the respondent in $6.41 \%$ $(n=5)$ of cases. Post-exposure prophylaxis was administered in $25.64 \%(n=20)$ of the cases reported (Table 1$)$.

Students reported, on a 1 to 10 scale, the degree of anxiety they felt after the accident using 1 to report no anxiety and 10 to report being very anxious. Twenty-six $(17.21 \%)$ of the respondents reported 10 as their level of anxiety after the incident; $11(7.28 \%)$ reported 1 . The mean level of anxiety among the injured students was $6.06(\mathrm{SD}+2.90)$.

A total of 151 participants reported an NSI and/or a mucocutaneous exposure. Overall, the number of accidents reported was as follows: $67.54 \%(n=102)$ of the participants who suffered an accident had had 1 event, $27.81 \%(n=42) 2$ to 3 events, and $4.63 \%(n=7)$ had experienced more than 3 events. Most of these accidents were not caused by or attributed to another health care worker ( $76.15 \%$ ).

As for the safety precautions during the incidents, $83.44 \%$ $(n=126)$ of the students were using gloves during the event. Re-capping used needles was reported as a typical maneuver by $74.68 \% \quad(n=233)$. Only $39.07 \% \quad(n=59)$ of the students were supervised while doing the procedure that caused the accident. Approximately a quarter (79/312) of the respondents were not familiar with reporting procedures in case of an NSI or a mucocutaneous exposure; only $61.5 \%$ had received information from their hospital about the standard protocol following a blood and body fluid exposure. Fifty nine percent (185/312) of the students would ask the resident on call for advice in case of an incident, $14.2 \%(45 / 312)$ would ask the attending physician, $4.49 \%(n=14)$ would ask a nurse, and $2.88 \%(\mathrm{n}=9)$ would ask a fellow student. In this survey, only $39.7 \%(n=124)$ of the students participating had completed the 3 doses of HBV vaccination.

\section{Discussion}

In this study, $34 \%$ of the students surveyed had suffered an NSI; similarly, $33 \%$ had experienced a mucocutaneous exposure. Thirty-seven percent $(81 / 218)$ of the students that were in their final year of medical school sustained at least one NSI during their medical studies. A previous study by Salzer and colleagues of medical students from the United Kingdom, Austria, and Germany reported an incidence of NSI in medical students in their final year of training at $34 \%$ and this was as high as $59 \%$ in the United States in graduate students. None of the mentioned studies, however, evaluated the number of procedures that the average medical student actually performs to assess the risk per procedure. ${ }^{2-7}$ In a similar study of 455 medical students from the United States, Kessler and colleagues registered a non-reporting rate of $82.9 \%$, which was considerably higher than the non-reporting rate of $48.34 \%$ in our study. ${ }^{3}$

A previous study conducted in the Hospital Universitario Dr. José Eleuterio Conzález during a five year period (2005-2011) reported that $45.42 \%$ of occupational exposures in healthcare workers occurred in medical students. In the same study, $87.7 \%$ of exposures were percutaneous injuries and $12.3 \%$ mucocutaneous exposures. ${ }^{8}$ In our study, medical students reported a similar number of NSIS $(n=110)$ and
Figure 1. Blood and Body Fluid Exposures by Year in Medical Students.

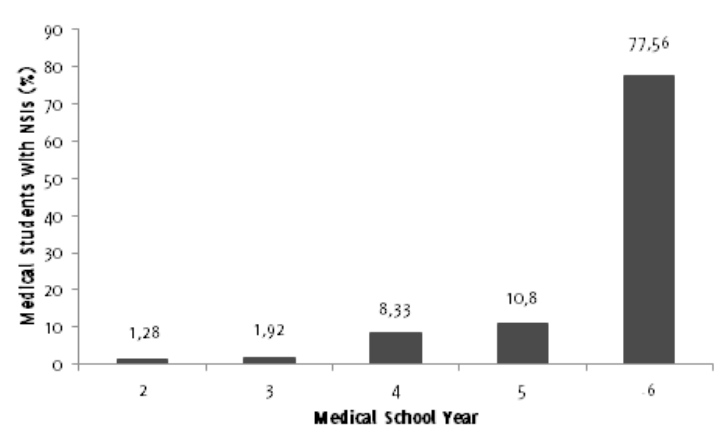

Table 1. Responses Provided by Medical Students on Reporting Procedures.

\begin{tabular}{lll}
\hline Characteristics & Frequency & $\%$ \\
\hline Did you report this accident? & & \\
\hline Yes & 78 & 51.66 \\
\hline No & 73 & 48.34 \\
\hline Was the source patient identified? & 73 & 93.58 \\
\hline Yes & 5 & 6.41 \\
\hline No & & \\
\hline Was the source patient already diagnosed with a & & \\
\hline HIV, HBV or HCV infection? & 8 & 10.25 \\
\hline Yes & 62 & 79.48 \\
\hline No & 8 & 10.25 \\
\hline Unknown & & \\
\hline Were serology tests performed on you after the & & \\
accident? & 46 & 58.97 \\
\hline Yes & 32 & 41.02 \\
\hline No & & \\
\hline Were serology tests performed on the source & & \\
\hline patient? & 60 & 76.92 \\
\hline Yes & 18 & 23.07 \\
\hline No & & \\
\hline Did you receive post-exposure prophylaxis? & 20 & 25.64 \\
\hline Yes & 58 & 74.35 \\
\hline No & & \\
\hline In & & \\
\hline
\end{tabular}

In case of a blood or body fluid exposure, do you know where to report the incident in your hospital?

\begin{tabular}{lcc} 
Yes & 233 & 74.68 \\
\hline No & 79 & 25.32 \\
$\begin{array}{l}\text { In case of a blood or body fluid exposure, who's } \\
\text { the first person you would ask for advice? }\end{array}$ & \\
Fellow student & 9 & 2.88 \\
Resident on call & 185 & 59.29 \\
Nurse & 14 & 4.49 \\
Attending physician & 59 & 14.42
\end{tabular}

mucocutaneous exposures $(n=106)$, suggesting that mucocutaneous exposures are more likely to go unreported, a finding similar to that in a study by Kessler and colleagues in the United States where $82.9 \%$ of the mucocutaneous exposures were not reported. ${ }^{3}$ We believe that the perception of a lower risk of acquiring a disease by mucocutaneous exposure than by NSIs is associated with lower reporting. 
As shown by French and Pakistani studies, an increase in the level of practice of universal precautions reduces the incidence of NSIs. ${ }^{9}{ }^{10}$ Salzer and colleagues indicate that education regarding NSIs may reduce the risk significantly. ${ }^{2}$ Although $63 \%$ (199/312) of the respondents attended at least one lecture about NSIs and mucocutaneous exposures, $74.68 \%$ reported recapping used needles as a usual maneuver, and $16 \%$ were not using gloves during the accident. This low compliance with universal precautions is probably due to the lack of continuous reminders and to human error.

Self-assessed knowledge of NSIs was rated as very good by only $5.1 \%$ of respondents. The results about knowledge of blood-borne pathogens was $100 \%$ for HIV and more than $90 \%$ for HBV and HCV; overall, however, the evaluation score of the population studied was low (average 3.6). This underscores the need for continuous reinforcement of safety and prevention measures during medical training and beyond.

Reporting of blood and body fluid exposures is critical because HBV immunoglobulin and vaccine should be administrated within the following days after a percutaneous exposure to HBsAg-positive blood, providing an estimated $85-95 \%$ protection from HBV infection. HIV prophylaxis should be started as soon as possible. ${ }^{5,1}$ In our study, only $39.7 \%$ (124) of the students had completed the three doses of HBV vaccination. Compared to results shown by Fica and colleagues in Chilean students where $98 \%$ of the students who suffered a NSI were vaccinated against HBV, in our survey there was a higher rate of susceptibility to become infected with HBV after a blood or body fluid exposure." In Mexico there is no law that obligates a healthcare worker to receive mandatory vaccination; thus, this in part explains the low compliance.
Self-reported level of anxiety was used to check the psychological impact of suffering a blood or body fluid exposure. This method could magnify the real level of anxiety among the students mainly because other variables such as personality and family stressors were not considered due the retrospective nature of the survey. Considering these limitations, a mean self-assessment of 6.1 on a scale of 1 to 10 is interesting because post-traumatic stress disorder in trainee doctors has been shown to be four times greater after an NSI. ${ }^{13}$

This survey studied a sample of students from different medical schools in Mexico. Although we cannot extrapolate our results to the whole Mexican medical student population, we consider our study to be a reflection of the general knowledge and need for improvement in education and prevention measures among Mexican medical students. The web link was distributed openly from person to person via e-mail and Facebook. This social network has been associated with a high daily usage among medical students $(64 \%)$ where the majority of medical students that use this network believe that they are equally active on Facebook as in real life. ${ }^{14}$ Thus we consider this method of distribution adequate for our survey.

We acknowledge our inability to calculate an accurate response (calculated to be $84.5 \%$ ) rate because of the nature of our survey and the possibility for a third person distribution, but we believe that the number of questionnaires answered can give an important perspective of the general knowledge that Mexican medical students and interns have regarding NSIs. 


\section{References}

1. Varsou 0, Lemon JS, Dick FD. Sharps injuries among medical students. Occup Med (Lond). 2009 0ct;59(7):509-11.

2. Salzer HJ, Hoenigl M, Kessler HH, Stigler FL, Raggam RB, Rippel KE, et al. Lack of risk-awareness and reporting behavior towards HIV infection through needle stick injury among European medical students. Int J Hyg Environ Health. 2011 Sep;214(5):407-10.

3. Kessler CS, McGuinn M, Spec A, Christensen J, Baragi R, Hershow RC. Underreporting of blood and body fluid exposures among health care students and trainees in the acute care setting: a 2007 survey. Am J Infect Control. $2011 \mathrm{Mar} ; 39(2): 129-34$.

4. Bernard JA, Dattilo JR, Laporte DM. The incidence and reporting of sharps exposure among medical students, orthopedic residents, and faculty at one institution. J Surg Educ. 2013 Sep-0ct;70(5): 660-8.

5. Centers for Disease Control and Prevention. Updated U.S. Public Health Service guidelines for the management of occupational exposures to HIV and recommendations for Postexposure Prophylaxis. MMWR 2005;54(No. RR9):1-17.

6. Sohn JW, Kim BG, Kim SH, Han C. Mental health of healthcare workers who experience needle stick and sharps injuries. J Occup Health. 2006 Nov;48(6):474-9.

7. Sharma GK, Gilson MM, Nathan H, Makary MA. Needle stick injuries among medical students: incidence and implications. Acad Med. 2009 Dec;84(12):1815-21.
8. Camacho-Ortiz A, Díaz-Rodríguez X, Rodríguez-López JM, Martínez-Palomares M, Palomares-De la Rosa A, Garza-González E. A 5-year surveillance of occupational exposure to bloodborne pathogens in a university teaching hospital in Monterrey, Mexico. Am J Infect Control. 2013 Sep; 41(9):e85-8. 9. Lamontagne F, Abiteboul D, Lolom I, Pellissier G, Tarantola A, Descamps JM, et al. Role of safety-engineered devices in preventing needle stick injuries in 32 French hospitals. Infect Control Hosp Epidemiol. 2007 Jan; 28(1):18-23. 10. Zafar A, Habib F, Hadwani R, Ejaz M, Khowaja K, Khowaja R, et al. Impact of infection control activities on the rate of needle stick injuries at a tertiary care hospital of Pakistan over a period of six years: an observational study. BMC Infect Di. 2009 May;9:78.

11. Centers for Disease Control and Prevention. Updated U.S. Public Health Service guidelines for the management of occupational exposures to HBV, HCV, and HIV and Recommendations for Postexposure Prophylaxis. MMWR 2001;50(No. RR-11):1-42.

12. Fica CA, Jemenao MI, Ruiz RG, Larrondo LM, Hurtado HC, Muñoz GG, et al. [Biological risk accidents among undergraduate healthcare students: five years experience]. Rev Chilena Infectol. 2010 Feb;27(1):34-9. Spanish.

13. Naghavi SH, Shabestari 0 , Alcolado J. Post-traumatic stress disorder in trainee doctors with previous needle stick injuries. Occup Med (Lond). 2013 Jun;63(4):260-5.

14. Farooqi H, Patel H, Aslam HM, Ansari IQ, Khan M, Iqbal N, et al. Effect of Facebook on the life of Medical University students. Int Arch Med. 2013 Oct;6(1):40.

\section{Acknowledgments}

None.

Conflict of Interest Statement at Funding

The Authors have no funding, financial relationships or conflicts of interest to disclose.

\section{Author Contributions}

Conception and design the work/idea: ACO, XDR, MMP. Collect data/obtaining results: XDR, RHG, MMP, RVV, IDP. Analysis and interpretation of data, Write the manuscript: ACO, RHG. Critical revision of the manuscript: ACO, ECG. Approval of the final version: ACO.

Cite as:

Camacho-Ortiz A, Díaz-Rodríguez X, Hernández-García R, Martínez-Palomares M, Chávez-Moreno S, Garza-Conzález E, et al. Exposure and Knowledge of Sharps Injuries by Medical Students in Seven States of Mexico. Int J Med Students. 2014 Nov-2015 Mar;3(1):24-8. 